\title{
A Switching Kinematic Model of an Octapedal Robot
}

\author{
Konstantinos Karydis, Ioannis Poulakakis and Herbert G. Tanner
}

\begin{abstract}
We propose a new horizontal locomotion model to describe the motion of an eight-legged bio-inspired miniature robot. The model does not include compliance, and can capture the kinematics of the observed locomotion behavior that corresponds to an alternating tetrapod gait. We exploit symmetries and synergies to reduce the eight-legged robot to a mechanism that is composed by two switching four-bar linkages, each representing the collective effect of a tetrapod in contact with the ground. Notwithstanding its apparent simplicity, the resulting model reproduces on average the motion of the robot. In addition, by properly tuning a family of physically-relevant parameters-including touchdown and sweep angles-different motion primitives corresponding to circular and forward motion can be realized. This model represents a first step toward developing reduced-order kinematic representations of legged robots that can be used for motion planning and feedback control purposes.
\end{abstract}

\section{INTRODUCTION}

A variety of small-scale crawling robots has been introduced in the last decade in an effort to realize the potential of such machines in applications involving searchand-rescue missions, building inspection, remote or hostile environment exploration, and others. This paper focuses on a novel octopedal robot-the OctoRoACH, see Fig. 1designed with the purpose of investigating the principles that govern reliable locomotion and proposing novel fabrication techniques that enable system integration at small scales.

Robots in this vein include the $\mathrm{HAMR}^{2}$ hexapod [1], which uses six piezo-electric actuators to drive its legs, and the Sprawlita [2] and iSprawl [3] robots that employ two actuators per leg and are capable of dynamic running motions by actively changing the leg kinematics. A different actuation approach based on a single motor governs the motion of the DASH [4] and RoACH [5], [6] robots. Along the same philosophy, DynaRoACH [7] extends the behaviors of its precursors [5], [6] by efficiently implementing dynamic maneuvers. However, its reliance on a single motor to control its alternating tripod mechanism leads to instabilities when turning at high yaw frequency on surfaces with moderate friction. This has been the motivation for introducing the eight-legged OctoRoACH platform [8].

This work focuses on modeling aspects. Motivated by research in sprawled arthropods [9], [10], a variety of reducedorder models have been proposed to explain the underlying similarity of the center-of-mass (COM) motion in animals and robots, despite their apparently diverse structural and morphological characteristics [11]. The majority of modeling efforts concentrates on simple spring-mass systems that rely

\footnotetext{
The authors are with the Department of Mechanical Engineering, University of Delaware. Email: \{kkaryd, poulakas, btanner\}@udel.edu
}

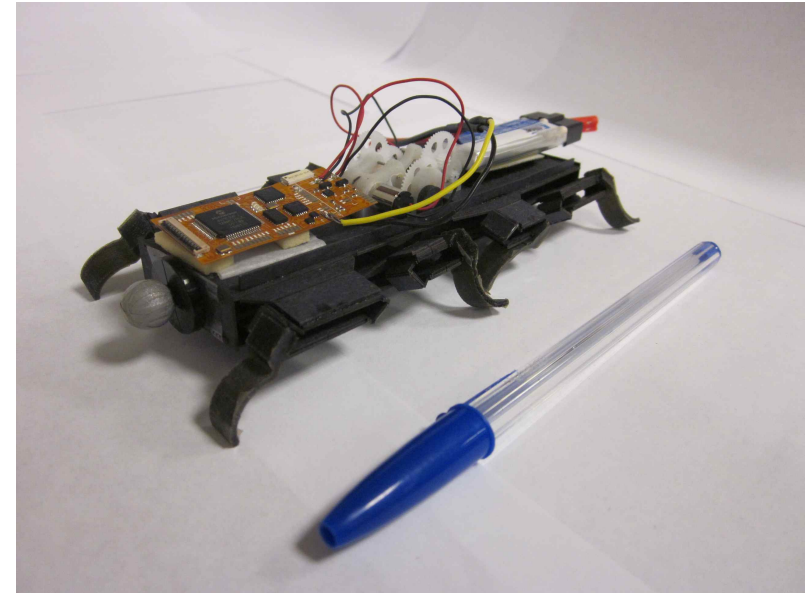

Fig. 1. The OctoRoaCH robot, designed by A. O. Pullin [8] at the University of California, Berkeley and manufactured by Motile Robotics, Inc. The robot has a total mass of $35 \mathrm{gm}$, its body size is $130 \times 60 \times 30 \mathrm{~mm}$ and the maximum speed it can reach is $0.5 \mathrm{~m} / \mathrm{s}$.

on a minimal set of variables and parameters to capture the salient features of a targeted gait behavior. Examples include the sagittal-plane Spring Loaded Inverted Pendulum (SLIP) [12], [13], and the horizontal-plane Lateral Leg Spring (LLS) models [14], [15]; see [16] for an extensive review.

In the context of hexapedal runners, the LLS model has proven its efficacy in explaining lateral stabilization [17], and in deriving turning strategies [18]. In its common configuration, the LLS is a conservative mechanical system composed by a rigid torso and two prismatic legs that are modeled as massless springs. Each leg represents the collective effect of a support tripod formed during stance by the front and rear ipsilateral ${ }^{1}$ and the contralateral middle legs that are in contact with the ground. Modeling octapedal crawlers like the OctoRoACH has received less attention in the relevant literature than hexapedal runners. Besides eight-legged models for crabs [19], which typically employ a metachronal tetrapod gait to move laterally in the horizontal plane, no further analyses appear to be available.

In this paper we introduce a kinematic model capable of capturing the motion of the OctoRoACH in the horizontal plane. Motivated by the structure of the mechanism-see [8] for a detailed description - the proposed model consists of two switching four-bar mechanisms, each representing an alternating tetrapod. Despite its simplicity, the mechanism generates forward and circular motions that correspond on average to the motion of the robot on the horizontal plane.

\footnotetext{
${ }^{1}$ Terminology: Ipsilateral means on the same side and contralateral means on the other side.
} 
Our goal is to use this model to develop motion planning and feedback control algorithms that will enable the OctoRoACH to operate as a member of a team that involves otherpossibly heterogeneous-robots.

We note here that first-order kinematic approximations have been used in the past to derive control laws for highly complex legged machines. For example, [20] employs a nonholonomic unicycle as a representation of the sixlegged robot RHex [21] to propose controllers for outdoor navigation guided by visual cues. The connection between the highly complex RHex and a unicycle model was further investigated in [22], where it was shown that a simplified version of the three-dimensional Newton-Euler dynamics of RHex can be reduced to such model when projected on the horizontal plane.

The structure of this paper is as follows. Section II briefly discusses the OctoRoACH and Section III introduces a model that captures the kinematics of the motion of the robot. Section IV analyzes the proposed model and Section V describes its behavior through simulations and discusses its ability to capture the motion of the robot. Lastly, Section VI concludes the paper.

\section{The OctoRoACH}

The OctoRoACH robot [8], shown in Fig. 1, is a miniature, bio-inspired robot designed and manufactured in a collaborative effort between the University of California, Berkeley and Motile Robotics, Inc. Like its six-legged predecessor, the Robotic Autonomous Crawling Hexapod (RoACH) [6], it was developed to explore the use of small-scale legged platforms as members of a team of robots that cooperate to achieve their task.

The robot consists of a rectangular body and eight legs that are organized so that ipsilateral legs are driven by a single actuator. No explicit coupling between the two sides is present, each of which is driven by a single DC brushed motor via a two-stage gear transmission. Changing the gains of the motors at each side results in either forward or circular motions. The robot features onboard electronics for communication and motor control, and a $300 \mathrm{mAh}$ lithium polymer ( $\mathrm{LiPo}$ ) battery powers the assembly; see [8] for a detailed account of the robot's design.

The leg drive kinematics combines a slider-crank linkage responsible for leg abduction and adduction, and a parallel four-bar mechanism responsible for leg protraction and retraction as shown in Fig. 2; a discussion of the mechanism can be found in [8]. As a result of its mechanical structure, the OctoRoACH implements a gait whose footfall pattern is composed by two alternating tetrapods as shown in Fig. 3. Such metachronal gaits have been studied in the context of an octapedal arthropod (the Ghost Crab) in [19], and are in direct analogy with the tripod gaits commonly employed by a variety of six-legged animals and robots; see [16].

Figs. 4(a) and 4(b) present typical paths of the physical robot, taken using a VICON motion capture system. The uncertainty inherent with ground contact-especially at this scale-manifests itself in the behavior of the robot, as is

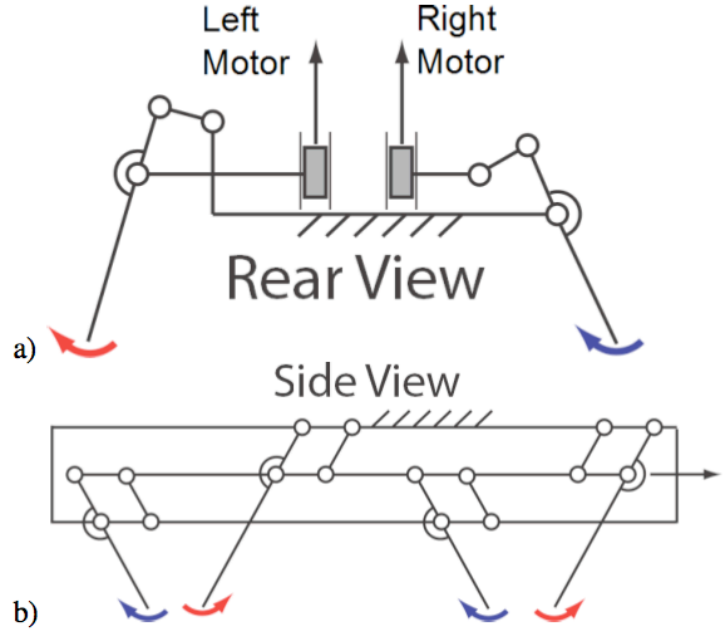

Fig. 2. Leg kinematics: a) The slider-crank linkage, responsible for the abduction and adduction motion (in and out motions from the sagittal plane) of the legs and b) the parallel four-bar mechanism which enables protraction and retraction of the legs. (Courtesy of A. O. Poullin; reproduced from [8] with the author's permission.)

readily seen in Figs. 4(a) and 4(b). Data in Fig. 4(a) correspond to inputs that should theoretically produce forward motion, and Fig. 4(b) to inputs that should in principle generate counter-clockwise circular motion. Capturing this type of uncertainty is part of ongoing research; the direction we are following is the introduction of stochasticity, but this falls outside the scope of the present paper. This paper specifically, treats the ideal (deterministic) case, and proposes a model that reproduces on average the robot's motion. Having a reasonable deterministic, and analytically tractable model of the robot is viewed as a first step toward further model refinements that include stochasticity.

\section{The Proposed Model}

In this section, a reduced-order, horizontal motion representation of the OctoRoACH is developed. Due to the robot's lightweight construction, we neglect dynamic effects and focus on kinematic analysis. The proposed model depicted at Fig. 5(a) consists of the robot's torso and eight legs that are modeled as non-deformable massless links attached at the torso via hip joints. In accordance to the footfall pattern of Fig. 3, the legs $\{1,2,3,4\}$ act in unison and form one tetrapod, and similarly the legs $\{5,6,7,8\}$ form the other tetrapod. Leg compliance along the horizontal direction is negligible for the platform of Fig. 1, and is not included in the model of Fig. 5(a). This is the main difference with the LLS model [14], [15], in which legs are modeled as massless

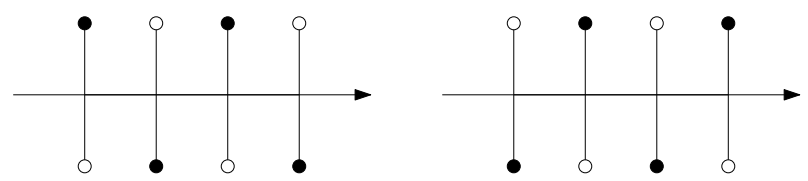

Fig. 3. The foot fall pattern of the robot which is a tetrapod gait. 


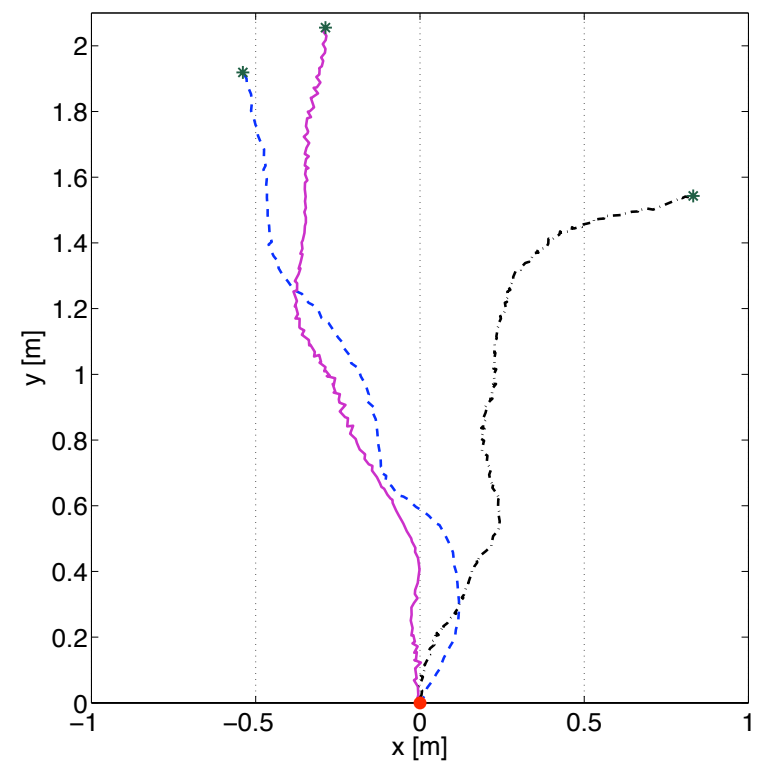

(a)

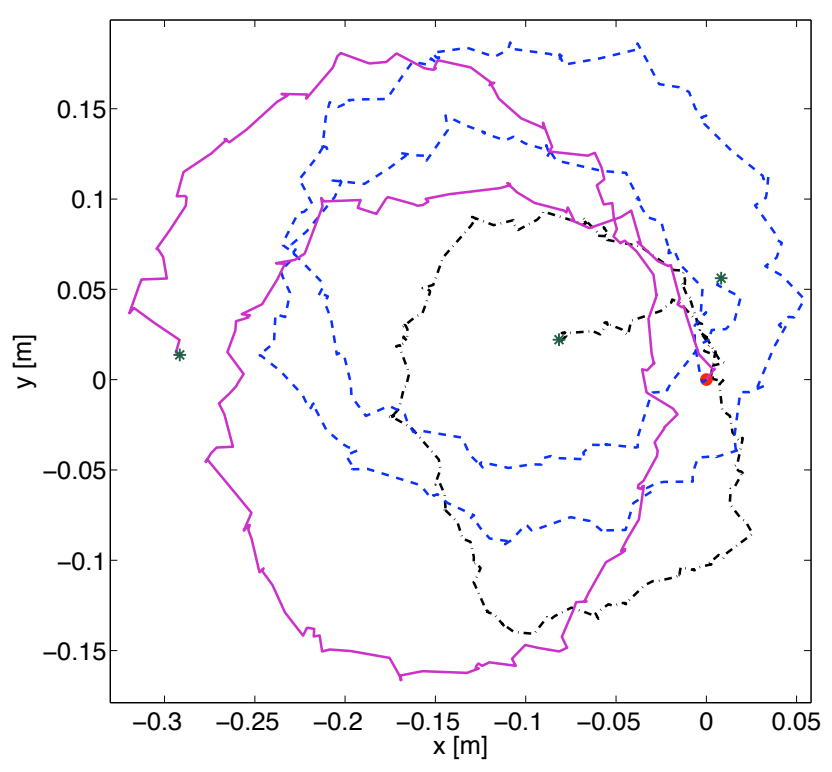

(b)

Fig. 4. Experimental results showing COM paths of the physical platform. In both experiments, the time duration was 20s. For each test, the starting point is shown with a red circle and the ending point with a green asterisk. (a) Motor gains that should produce forward motion. The dashed blue line corresponds to motor gains $\{225,225\}$, the solid magenta to $\{150,150\}$ and the dashed-dot black line to $\{100$, 100$\}$. (b) Motor gains that should produce motion along a counter-clockwise circular path. All tests start at the same location and orientation and the motors gains are the same $\{25,75\}$.

springs.

The configuration variables of this model consist of the Cartesian position $(x, y)$ of the geometric center $G$ of the platform's body with respect to an inertial coordinate frame $O$, and the angle $\theta$ between the longitudinal body-frame axis and the vertical axis of the inertia frame; see Fig. 5(a). Intuitively, the mechanism of Fig. 5(a) propels itself in the horizontal plane through a "rowing" motion.

To reduce the model, we make use of symmetries and

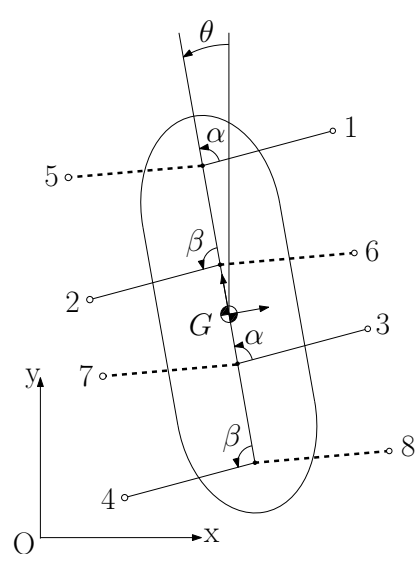

(a)

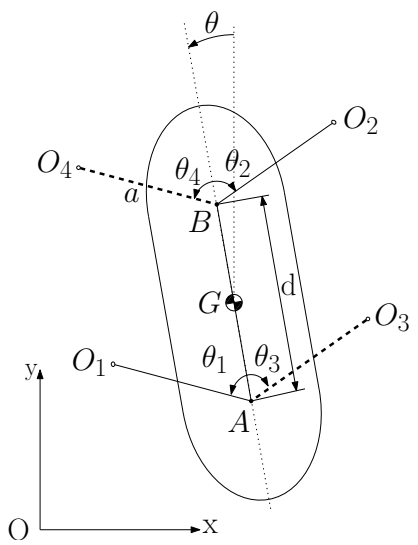

(b)
Fig. 5. (a) A kinematic octapedal model for the OctoRoACH in the horizontal plane. (b) The abstracted model in which each of the two tetrapods is replaced by a pair of legs: the tetrapod $\{1,2,3,4\}$ corresponds to the legs $\left\{O_{1}, O_{2}\right\}$ (right pair) and the tetrapod $\{5,6,7,8\}$ to the legs $\left\{\mathrm{O}_{3}, \mathrm{O}_{4}\right\}$ (left pair). Notice that when the right pair is active, the mechanism defined by the links $\left\{O_{1} A, A B, B O_{2}\right\}$ corresponds to a fourbar linkage. Similarly, when the left pair is active, the mechanism defined by the links $\left\{\mathrm{O}_{3} \mathrm{~A}, \mathrm{AB}, \mathrm{BO}_{4}\right\}$ corresponds to a four-bar linkage. synergies that govern the motion of the legs. In particular, the ipsilateral legs of each tetrapod touch the ground at the same instant, rotate with the same angular velocity and move in phase, maintaining the same angle with respect to the longitudinal body-frame axis. For example, with reference to Fig. 5(a), both legs in the pair $\{1,3\}$ form the same angle $\alpha$ with respect to the body axis as the system moves forward, corresponding to a coupled in-phase motion of these legs. The complementary legs $\{2,4\}$ in the same tetrapod move in the same way forming an angle $\beta$ with the body axis, which in general can be different from $\alpha$. A similar motion takes place when the other tetrapod is active.

Based on these observations, we can combine each pair of ipsilateral legs in an active tetrapod into a single "virtual" leg that induces the same displacement on the mechanism. The virtual leg for each ipsilateral pair will be located at a properly selected point along the body-frame longitudinal axis. Note that the initial condition and the range of motion of a virtual leg can be different compared to the angular motion of the legs in the pair it replaces.

This abstraction leads to the four-legged kinematic model depicted in Fig. 5(b). In this model, the contralateral virtual legs, e.g. $\left\{O_{1}, O_{2}\right\}$ for the right ${ }^{2}$ pair, represent the collective effect of the tetrapod, e.g. $\{1,2,3,4\}$, respectively. Notice that contralateral legs in a pair of virtual legs can form different angles and angular velocities with respect to the longitudinal body-frame axis, e.g., $\theta_{1} \neq \theta_{2}$ and $\dot{\theta}_{1} \neq \dot{\theta}_{2}$ in general for $\left\{\mathrm{O}_{1}, \mathrm{O}_{2}\right\}$ in Fig. 5(b). However, both pairs $\left(\theta_{1}, \theta_{2}\right)$ and $\left(\dot{\theta}_{1}, \dot{\theta}_{2}\right)$, are coupled through the equations of the closed-loop mechanism formed between points $O_{1}, A, B$,

\footnotetext{
${ }^{2}$ Convention: With reference to Fig. 5(b), $\left\{\mathrm{O}_{1}, \mathrm{O}_{2}\right\}$ is referred to as the right pair since the right leg $\mathrm{O}_{2}$ is the leading leg.
} 
and $\mathrm{O}_{2}$; see Fig. 6. This coupling is the subject of the section that follows. It is only mentioned here that the resulting motion corresponds to the motion produced by a switching four-bar linkage.

\section{Analysis of the Abstracted Model}

In this section, the kinematics of the mechanism introduced in Section III is derived. In the analysis that follows, a $50 \%$ duty cycle for the two pairs $\left\{O_{1}, O_{2}\right\}$ and $\left\{O_{3}, O_{4}\right\}$ will be assumed. At any given time instant, only one pair is in contact with the ground.

As mentioned in Section III, during each stance phase, when a pair $\left\{O_{i}, O_{i+1}\right\}$ is in contact with the ground, the system corresponds to a four-bar mechanism [23]. The motion of the four-bar linkage is determined by a single degree of freedom, which we take to be the angle $\theta$ in Fig. 6-all other variables $\left(x, y, \theta_{1}, \ldots, \theta_{4}\right)$ can be expressed in terms of this angle $\theta$. Switching between tetrapods gives rise to a switched-mode system [24],

$$
\dot{\theta}=f_{\alpha}(\theta), \quad t \in\left[\tau_{i-1}, \tau_{i}\right], \quad i=1, \ldots, N \in \mathbb{N}
$$

where $\tau_{i}$ are switching times (time instants at the beginning and end of duty cycles), $i$ is the index of a switching instant, $\alpha=\{1,2\}$ is the modal index of the mode in which the system is in (left or right pair on the ground), and $f_{\alpha}$ is the modal function that determines the kinematics of the system during that phase. At switching times, the normal time derivative is either replaced by the appropriate one side derivative or can take the form of a differential inclusion.

The modal functions $f_{\alpha}$ can be determined directly from the vector-loop equations [23] of the four-bar mechanism shown in Fig. 6. In the representation of Fig. 6, points $O_{1}$ and $\mathrm{O}_{2}$ are considered fixed, implying that the angle $\phi_{s}$ is

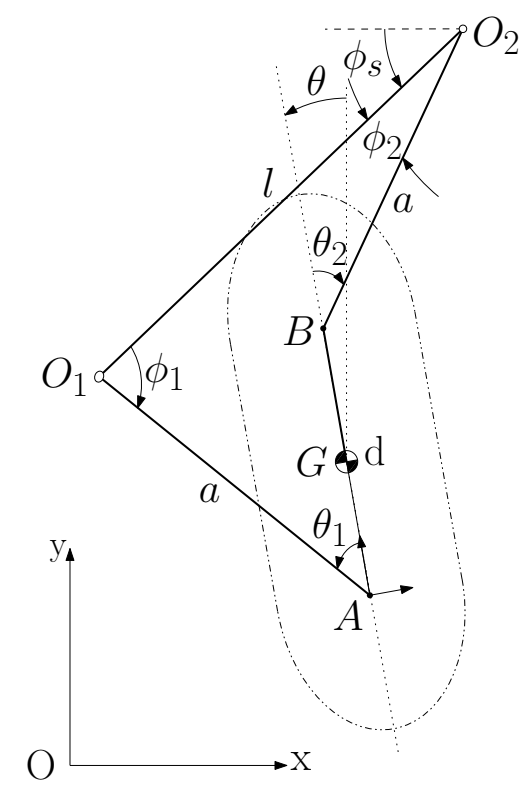

Fig. 6. Kinematic analysis of the four-bar mechanism that describes the problem. $a, d$ are parameters that define the size of the mechanism, $O_{1}, O_{2}$ and $\phi_{s}$, after defined, remain fixed for the rest of the sweeping phase. constant, while $\theta_{1}$ represents the independent variable. The (position) vector-loop equation is

$$
\boldsymbol{R}_{A O_{1}}+\boldsymbol{R}_{O_{1} O_{2}}-\boldsymbol{R}_{A B}-\boldsymbol{R}_{B O_{2}}=0 .
$$

Using the exponential representation of the participating vectors in (2), we obtain,

$$
\begin{aligned}
a e^{j\left(\pi / 2+\theta_{1}\right)}+l e^{j\left(\phi_{1}-\pi / 2+\theta_{1}\right)} & \\
& -d e^{j \pi / 2}-a e^{j\left(\pi / 2-\theta_{2}\right)}=0 .
\end{aligned}
$$

Then, by $e^{ \pm j \theta}=\cos \theta \pm j \sin \theta$ through separating real and imaginary parts we have

$$
\begin{aligned}
-a \sin \left(\theta_{1}\right)+l \sin \left(\phi_{1}+\theta_{1}\right)-a \sin \left(\theta_{2}\right) & =0 \\
a \cos \left(\theta_{1}\right)+l \cos \left(\phi_{1}+\theta_{1}\right)-d-a \cos \left(\theta_{2}\right) & =0 .
\end{aligned}
$$

The geometry of the mechanism also implies

$$
\begin{array}{ll}
\theta_{1}+\phi_{1}+\theta_{2}+\phi_{2}=\pi & \text { if } A B \cap O_{1} O_{2}=\emptyset \\
\theta_{1}+\theta_{2}+\phi_{1}=\pi+\phi_{2} & \text { if } A B \cap O_{1} O_{2} \neq \emptyset
\end{array}
$$

depending on whether the line segments $A B$ and $O_{1} O_{2}$ intersect. Equations (4)-(5) then describe a family of angles parameterized through $\theta_{1}$. An explicit solution for $\theta_{1}$, is difficult to obtain due to the presence of trigonometric functions in (4). Note however, that when taking time derivatives in (4)-(5) the angular velocities will appear linearly, allowing the direct determination of the modal functions in (1).

\section{Simulation Results}

This section uses the mechanism described above to reproduce, on average, the forward and circular motion primitives of the OctoRoACH. The parameters of the model are chosen first. In Fig. 6, $d$ is the distance between the two virtual hip joints $A$ and $B$; based on the physical robot's body we obtain $d=5 \mathrm{~cm}$. On the other hand, the virtual leg length, $a$, does not correspond directly to the robot and it is determined by matching experimental data and simulation results as $a=2 \mathrm{~cm}$; this is similar to what was done in [17].

In the sequel, $\theta_{i}^{\text {td }}$ for $i=1, \ldots, 4$, denote the touchdown angles of the virtual legs in the model of Fig. $6, \theta_{i}^{\text {td }} \geq$ 0 , and $\boldsymbol{x}_{G} \boldsymbol{x}_{O_{i}}$ the position coordinates of points $G$ and $O_{i}$, respectively. Let $\psi_{i}$ denote the range (sweep angle) of each $\theta_{i}$, as the corresponding virtual leg rotates around its hip point, from the configuration where it first touches the ground-marked by $\theta_{i}^{\text {td }}$ - until it looses contact at lift-off; note that $\psi_{i}=\pi-\theta_{i}^{\text {td }}$ represents a full sweep.

Due to symmetry, the motion of the left pair of virtual legs is also given by (4) and (5) - one just needs to substitute $\left\{\theta_{1}, \theta_{2}, \phi_{1}, \phi_{2}\right\}$ for $\left\{\theta_{3}, \theta_{4}, \phi_{3}, \phi_{4}\right\}$. Table I describes the steps required to numerically simulate the model (4)-(5), under the assumption of a $50 \%$ duty cycle.

Fig. 7 depicts the evolution of angles $\theta_{1}$ and $\theta_{2}$, during a single stride of the right leg pair starting at different touchdown angles; the results refer to the full sweep case. Clearly, a linear increase on $\theta_{1}$ has a nonlinear effect on $\theta_{2}$ as expected based on the geometry of the mechanism. Note however that the two angles agree at the beginning and end of the stride, when full sweep motion is implemented. 
TABLE I

SIMULATION PROCESS FOR ONE CYCLE

\section{Give: $\theta, \boldsymbol{x}_{G}$;}

2. Give: $\theta_{1}^{\text {td }}, \theta_{2}^{\text {td }}$ for the right pair;

3. Calculate and fix: $\boldsymbol{x}_{O_{1}}, \boldsymbol{x}_{O_{2}}, l, \phi_{s}$;

4. Solve (4)-(5) as $\theta_{1}$ evolves;

5. Calculate $\theta, \boldsymbol{x}_{G}$ at end of stride;

6. Give: $\theta_{3}^{\text {td }}, \theta_{4}^{\text {td }}$ for the left pair;

7. Calculate and fix: $\boldsymbol{x}_{O_{3}}, \boldsymbol{x}_{O_{4}}, l, \phi_{s}$;

8. Solve (4)-(5) as $\theta_{3}$ evolves;

9. Calculate $\theta, \boldsymbol{x}_{G}$ at end of stride.

Finally, it is remarked that increasing the touchdown angles $\theta_{1}^{\mathrm{td}}=\theta_{2}^{\mathrm{td}}$, reduces the range of motion of the legs during a full sweep; see Fig. 7.

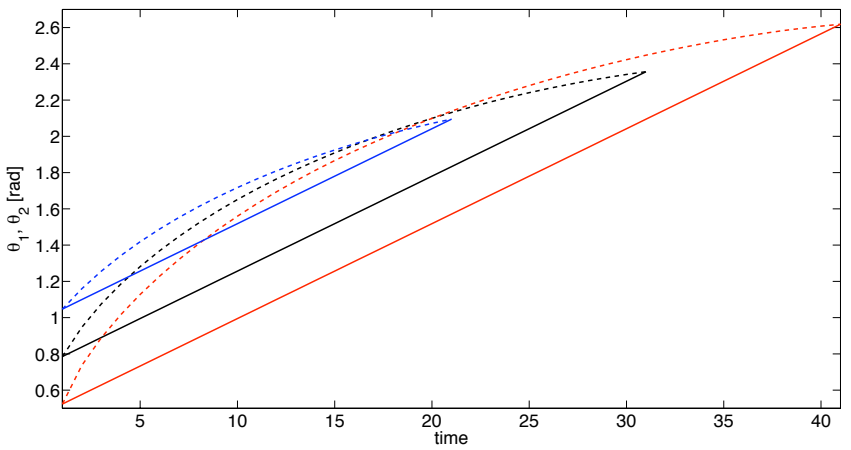

Fig. 7. Evolution of $\theta_{1}$ and $\theta_{2}$ for full stride for different values of the touchdown angles $\theta_{1}^{\text {td }}=\theta_{2}^{\text {td }}$. Solid lines correspond to $\theta_{1}$ and dashed ones to $\theta_{2}$. The red lines are for the case $\theta_{1}^{\mathrm{td}}=\theta_{2}^{\mathrm{td}}=\pi / 6$, the black for $\theta_{1}^{\text {td }}=\theta_{2}^{\text {td }}=\pi / 4$ and the blue for $\theta_{1}^{\text {td }}=\theta_{2}^{\text {td }}=\pi / 3$.

\section{A. Reproducing forward motion}

To reproduce a forward motion of the robot, all legs operate in a full sweep mode. In Fig. 8, the simulated trajectory of point $G$ is plotted for different touchdown angles, and for the case of a full sweep under a $50 \%$ duty cycle. The results show that the alternating tetrapod gait can generate forward motion in the proposed kinematic model. All the paths that have been produced exhibit oscillations along the $x$ direction, which in some cases are more pronounced depending on the touchdown angles. Fig. 8(a) indicates that, as the touchdown angle increases, the total length traveled decreases and the amplitude of the oscillation observed also decreases. The experimental results presented in Fig. 4(a), although very noisy, confirm the oscillatory motion predicted by the model.

As mentioned above, the amplitude of the oscillation and the total length of the path traversed are related: increasing the touchdown angles reduces both the length of the path and the amplitude of the corresponding oscillations. Fig. 9 also illustrates this fact. The different curves in Fig. 9 are associated with different values of touchdown angles, and show that the oscillation in the yaw angle $\theta$ as a function of the distance traveled along the $y$ inertial frame axis.

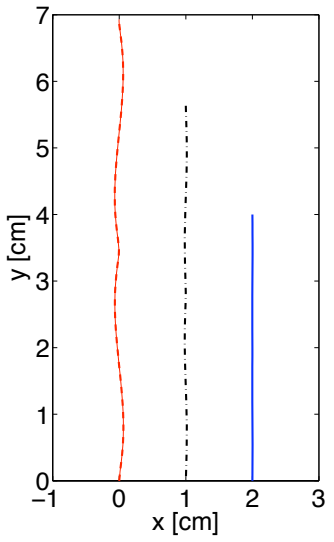

(a)

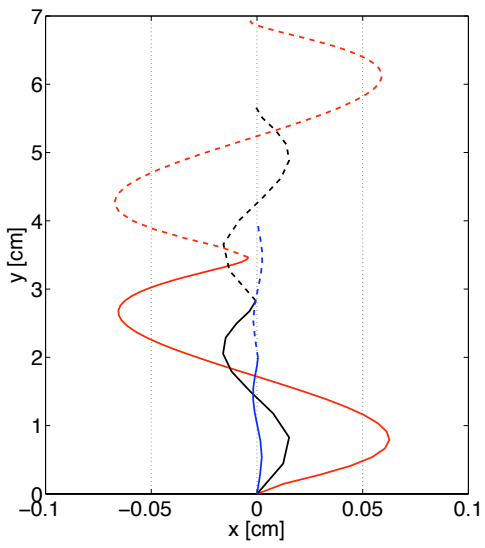

(b)
Fig. 8. (a) Forward motion for different touchdown angles. (b) Magnified view to capture the oscillations observed during the motion. Three cases are shown: the dashed (red) path corresponds to $\theta_{i}^{\text {td }}=\pi / 6$, the dashed-dot (black) to $\theta_{i}^{\text {td }}=\pi / 4$ and the solid (blue) to $\theta_{i}^{\text {td }}=\pi / 3, i=1, \ldots, 4$.

Clearly, larger touchdown angles-in full sweep moderesult to smaller sweep angles, which are directly related to the displacement for geometric center $G$ of the robot's body. Intuitively, the situation corresponds to rowing with shorter strokes.

\section{B. Reproducing circular paths}

The model offers several parameters that can be adjusted to produce different motion profiles; principal among them are the touchdown angles, the sweep angles, and the duty cycle. A particular combination of parameters that produces circular motion in this model corresponds to only one virtual leg pair used for propulsion, while the other, when it is on the ground, is assumed to be stationary and merely provides support. In this case, (1) still describes the switching between the two modes, where one of them corresponds to a modal function $f_{\alpha}$ that is set to zero.

Fig. 10 shows the path of point $G$, for different touchdown angle settings, and under the assumption that only the right

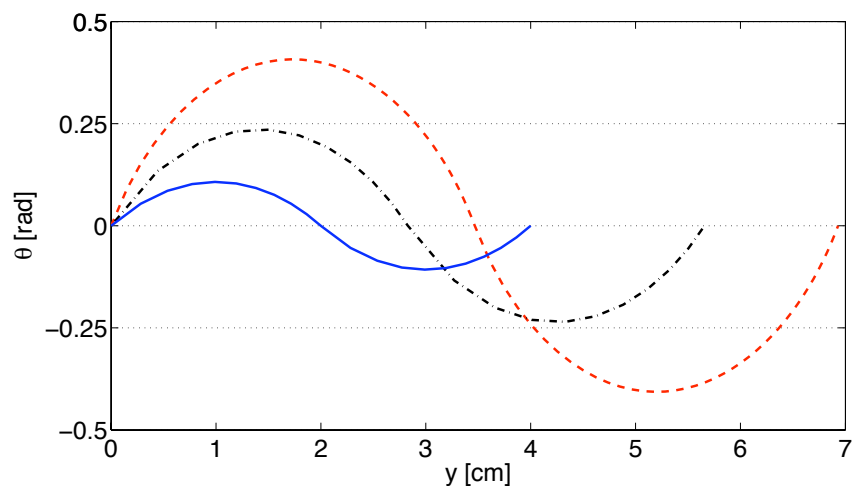

Fig. 9. Evolution of the orientation (yaw) angle $\theta$ as a function of the distance traveled along the inertial $y$-axis. The different curves correspond to simulation with different values of angles $\theta_{i}^{\text {td }}$. The figure suggests that an increase on $\theta_{i}^{\text {td }}$ leads to more oscillatory behavior (larger amplitude with smaller frequency) in terms of $\theta$. 


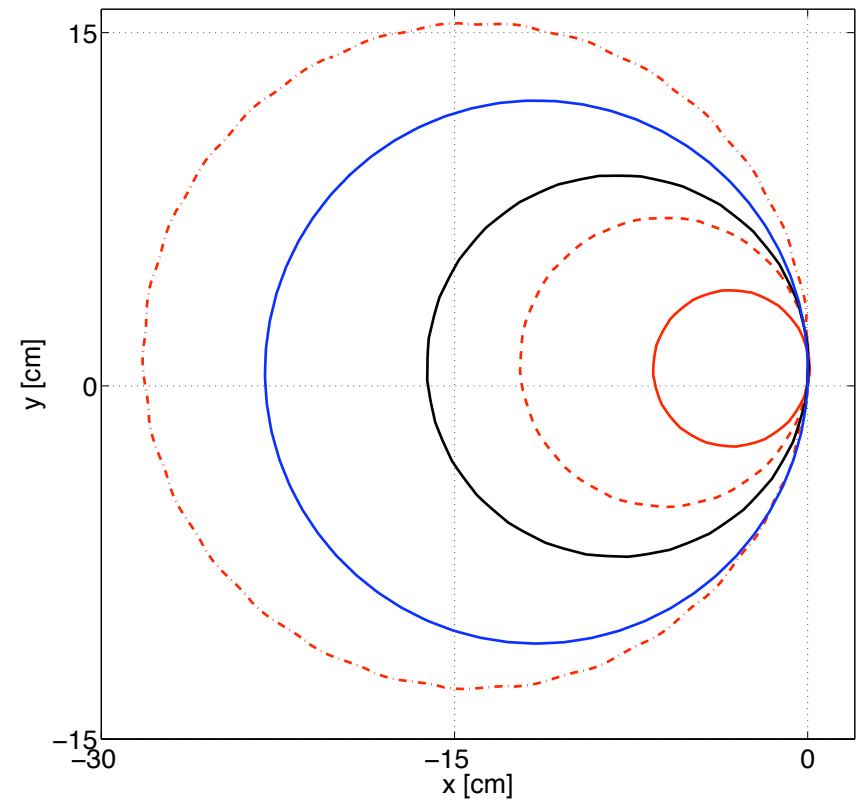

Fig. 10. Circular paths for different touchdown and sweep angles. The solid red line corresponds to $\theta_{1}^{\text {td }}=\theta_{2}^{\text {td }}=\pi / 6$, the black line to $\theta_{1}^{\text {td }}=\theta_{2}^{\text {td }}=$ $\pi / 4$ and the blue one to $\theta_{1}^{\text {td }}=\theta_{2}^{\text {td }}=\pi / 3$. Fixing $\theta_{1}^{\text {td }}=\theta_{2}^{\text {td }}=\pi / 6$, the red (smallest) solid curve is produced when $\psi=\pi / 6$, the dashed red curve when $\psi=\pi / 3$ and the dashed dotted one when $\psi=\pi / 2$.

pair of legs is moving, while the left pair, when active, only provides support. The initial position is the same for all cases. We notice that the radius of the circular path increases as the touchdown angle or, equivalently, the sweep angle increases. As a result, sharper turns can be achieved by decreasing the touchdown angles; that is, with smaller "steps" the mechanism can achieve smaller turning radii.

\section{CONClusions AND Future Work}

The horizontal motion of an eight-legged arthropod robot, the OctoRoACH, can be represented by a new kinematic model with stick legs. Motivated by the transmission system of the robot, a switching four-bar mechanism that implements an alternating tetrapod gait is introduced and analyzed. The proposed model offers a number of parameters that can be independently adjusted to produce different motion primitives, including forward and circular motion, in a way that reproduces the deterministic behavior of the platform. Ongoing work seeks to extend this model to capture the inherent stochasticity in the behavior of the robot, as characteristically seen in the path plots of Figs. 4(a) and 4(b). In the context of the OctoRoACH, a large part of the uncertainty is introduced through inexact ground contact and a stochastic model that captures this effect will be more appropriate to describe the experimentally observed behavior of the robot.

\section{REFERENCES}

[1] A. T. Baisch, P. Sreetharan, and R. J. Wood, "Biologically-inspired locomotion of a $2 \mathrm{~g}$ hexapod robot." in IROS'10, 2010, pp. 5360-5365.
[2] J. G. Cham, S. A. Bailey, J. E. Clark, R. J. Full, and M. R. Cutkosky, "Fast and Robust: Hexapedal Robots via Shape Deposition Manufacturing," The International Journal of Robotics Research, vol. 21, no. 10-11, pp. 869-882, 2002.

[3] S. Kim, J. E. Clark, and M. R. Cutkosky, "iSprawl: Design and Tuning for High-speed Autonomous Open-loop Running," The International Journal of Robotics Research, vol. 25, no. 9, pp. 903-912, Sep. 2006.

[4] P. Birkmeyer, K. Peterson, and R. S. Fearing, "Dash: A dynamic $16 \mathrm{~g}$ hexapedal robot," in 2009 IEEE/RSJ International Conference on Intelligent Robots and Systems, October 11-15, 2009, St. Louis, MO, USA. IEEE, 2009, pp. 2683-2689.

[5] A. M. Hoover, E. Steltz, and R. S. Fearing, "Roach: An autonomous $2.4 \mathrm{~g}$ crawling hexapod robot," in 2008 IEEE/RSJ International Conference on Intelligent Robots and Systems, September 22-26, 2008, Acropolis Convention Center, Nice, France. IEEE, 2008, pp. 26-33.

[6] C. Li, A. M. Hoover, P. Birkmeyer, P. B. Umbanhowar, R. S. Fearing, and D. I. Goldman, "Systematic study of the performance of small robots on controlled laboratory substrates," in Micro- and Nanotechnology Sensors, Systems, and Applications II, Proc of the SPIE Conf on. SPIE, Apr. 2010, pp. 76 790Z-76 790Z-13.

[7] A. M. Hoover, S. Burden, X.-Y. Fu, S. Shankar Sastry, and R. S. Fearing, "Bio-inspired design and dynamic maneuverability of a minimally actuated six-legged robot," Sep. 2010, pp. 869-876.

[8] A. Pullin, N. Kohut, D. Zarrouk, and R. Fearing, "Dynamic turning of $13 \mathrm{~cm}$ robot comparing tail and differential drive," in IEEE Int. Conf. Robotics and Automation, may 2012, to appear.

[9] R. Blickhan and R. J. Full, "Similarity in multilegged locomotion: Bouncing like a monopode," Journal of Comparative Physiology A: Neuroethology, Sensory, Neural, and Behavioral Physiology, vol. 173, pp. 509-517, 1993.

[10] G. A. Cavagna, N. C. Heglund, and C. R. Taylor, "Mechanical work in terrestrial locomotion: two basic mechanisms for minimizing energy expenditure," Am J Physiol Regulatory Integrative Comp Physiol, vol. 233, pp. 243-261, 1977.

[11] R. Full and D. Koditschek, "Templates and anchors: Neuromechanical hypotheses of legged locomotion on land," in The Journal of Experimental Biology, vol. 202, 1999, pp. 3325-3332.

[12] W. J. Schwind, "Spring Loaded Inverted Pendulum Running: A Plant Model," Ph.D. dissertation, University of Michigan, 1998.

[13] I. Poulakakis and J. W. Grizzle, "Modeling and Control of the Monopedal Running Robot Thumper," in Proceedings of the IEEE International Conference on Robotics and Automation, Kobe, Japan, 2009, pp. 3327-3334.

[14] J. Schmitt and P. Holmes, "Mechanical models for insect locomotion: dynamics and stability in the horizontal plane I. Theory," Biological Cybernetics, vol. 83, no. 6, pp. 501-515, Nov. 2000.

[15] _ "Mechanical models for insect locomotion: dynamics and stability in the horizontal plane - II. Application," Biological Cybernetics, vol. 83 , no. 6 , pp. $517-527$, Nov. 2000.

[16] P. Holmes, R. J. Full, D. E. Koditschek, and J. Guckenheimer, "The dynamics of legged locomotion: Models, analyses, and challenges," 2006.

[17] J. E. Seipel, P. J. Holmes, and R. J. Full, "Dynamics and stability of insect locomotion: a hexapedal model for horizontal plane motions," Biological Cybernetics, vol. 91, no. 2, pp. 76-90, 2004.

[18] J. Proctor and P. Holmes, "Steering by Transient Destabilization in Piecewise-Holonomic Models of Legged Locomotion," Regular and Chaotic Dynamics, vol. 13, no. 4, pp. 267-282, 2008.

[19] R. Blickhan and R. J. Full, "Locomotion energetics of ghost crab. ii. mechanics of the center of mass during walking and running," Journal of Experimental Biology, vol. 130, no. 1, pp. 155-174, 1987.

[20] G. A. Lopes and D. E. Koditschek, "Visual servoing for nonholonomically constrained three degree of freedom kinematic systems," The International Journal of Robotics Research, vol. 26, no. 7, pp. 715736, 2007.

[21] U. Saranli, M. Bühler, and D. E. Koditschek, "RHex: A Simple and Highly Mobile Hexapod Robot," The International Journal of Robotics Research, vol. 20, no. 7, pp. 616-31, Jul. 2001.

[22] D. Panagou and H. Tanner, "Modeling of a hexapod robot; kinematic equivalence to a unicycle," University of Delaware, Tech. Rep. UDMETR-2009-001, 2009.

[23] R. Norton, Machine Design. Prentice Hall, 2010.

[24] M. Egerstedt, Y. Wardi, and H. Axelsson, "Transition-time optimization for switched-mode dynamical systems," IEEE Transactions on Automatic Control, vol. 51, no. 1, pp. 110-115, 2006. 